\title{
Modeling Computer Virus and Its Dynamics
}

\author{
Mei Peng, ${ }^{1}$ Xing He, ${ }^{2}$ Junjian Huang, ${ }^{3}$ and Tao Dong ${ }^{4}$ \\ ${ }^{1}$ College of Mathematical and Computer Science, Yangtze Normal University, Chongqing 400084, China \\ ${ }^{2}$ College of Computer Science, Chongqing University, Chongqing 400030, China \\ ${ }^{3}$ School of Computer Science, Chongqing University of Education, Chongqing 400067, China \\ ${ }^{4}$ College of Software and Engineering, Chongqing University of Posts and Telecommunications, Chongqing 400065, China
}

Correspondence should be addressed to Mei Peng; pmgs@qq.com

Received 27 March 2013; Accepted 9 June 2013

Academic Editor: Tingwen Huang

Copyright (C) 2013 Mei Peng et al. This is an open access article distributed under the Creative Commons Attribution License, which permits unrestricted use, distribution, and reproduction in any medium, provided the original work is properly cited.

Based on that the computer will be infected by infected computer and exposed computer, and some of the computers which are in suscepitible status and exposed status can get immunity by antivirus ability, a novel coumputer virus model is established. The dynamic behaviors of this model are investigated. First, the basic reproduction number $R_{0}$, which is a threshold of the computer virus spreading in internet, is determined. Second, this model has a virus-free equilibrium $P^{0}$, which means that the infected part of the computer disappears, and the virus dies out, and $P^{0}$ is a globally asymptotically stable equilibrium if $R_{0}<1$. Third, if $R_{0}>1$ then this model has only one viral equilibrium $P^{*}$, which means that the computer persists at a constant endemic level, and $P^{*}$ is also globally asymptotically stable. Finally, some numerical examples are given to demonstrate the analytical results.

\section{Introduction}

Computer virus is a malicious mobile code which including virus, Trojan horses, worm, and logic bomb. It is a program that can copy itself and attack other computers. And they are residing by erasing data, damaging files, or modifying the normal operation. Due to the high similarity between computer virus and biological virus [1], various computer virus propagation models are proposed [2-4]. This dynamical modeling of the spread process of computer virus is an effective approach to the understanding of the behavior of computer viruses because on this basis, some effective measures can be posed to prevent infection.

The computer virus has a latent period, during which individuals are exposed to a computer virus but are not yet infectious. An infected computer which is in latency, called exposed computer, will not infect other computers immediately; however, it still can be infected. Based on these characteristics, delay is used in some models of computer virus to describe that although the exposed computer does not infect other computers, it still has infectivity $[5,6]$. Yang et al. $[7,8]$ proposed an SLB and SLBS models; in these models, the authors considered that the computer virus has latency, and the computer also has infectivity in the period of latency. However, they do not show the length of latency and take into account the impact of artificial immunization ways such as installing antivirus software. And the newly entered in the internet from the susceptible status to exposed status, the contact rate is the same as that of susceptible status entering into infected status. In this paper, a novel model of computer virus, known as SEIR model, is put forward to describe the susceptible computer which can be infected by the other infected or exposed computer and come into the exposed status. In the SEIR model, based on artificial immunity, we consider the bilinear incidence rate for the latent and infection status. Assume that the computers which newly entered the internet are susceptible, the computers correspond with exposed computers, and their adequate contact rate is denoted by $\beta_{1}$, and computers also correspond with infected computers, and their adequate contact rate is denoted by $\beta_{2}$. So, the fraction of the computer which newly entered the internet will enter the class $R$ by anti-virus software; the fraction of computers contact with exposed and infected computer will stay latent before becoming infectious and enter the class $E$. It is shown that the dynamic behavior of the proposed model is determined by a threshold $R_{0}$, and this 
model has a virus-free equilibrium $P^{0}$, and $P^{0}$ is a globally asymptotically stable equilibrium if $R_{0}<1$; if $R_{0}>1$ this model has only one viral equilibrium $P^{*}$, and it is globally asymptotically stable.

This paper is organized as follows. Section 2 formulates a novel computer virus mode. Section 3 proves the global stability of the virus-free equilibrium. Section 4 discusses the stability of the viral equilibrium. In Section 5, numerical simulations are given to present the effectiveness of the theoretic results. Finally, Section 6 summarizes this work.

\section{Model Formulation}

At any time, a computer is classified as internal and external depending on weather it is connected to internet or not. At that time, all of the internet computers are further categorized into four classes: (1) susceptible computers, that is, uninfected computers and new computers which connected to network; (2) exposed computers, that is, infected but not yet broken-out; (3) infectious computers; (4) recovered computers, that is, virus-free computer having immunity. Let $S(t), E(t), I(t), R(t)$ denote their corresponding numbers at time $t$, without ambiguity; $S(t), E(t), I(t), R(t)$ will be abbreviated as $S, E, I, R$, respectively. The model is formulated as the following system of differential equations:

$$
\begin{gathered}
S^{\prime}=(1-p) N-\beta_{1} S I-\beta_{2} S E-p S-\mu S, \\
E^{\prime}=\beta_{1} S I+\beta_{2} S E-k E-\alpha E-\mu E, \\
I^{\prime}=\alpha E-r I-\mu I, \\
R^{\prime}=p S+k E+r I, \\
N(t)=S(t)+E(t)+I(t)+R(t) .
\end{gathered}
$$

We may see that the first three equations in (1) are independent of the fourth equation, and therefore, the fourth equation can be omitted without loss of generality. Hence, system (1) can be rewritten as

$$
\begin{gathered}
S^{\prime}=A-\beta_{1} S I-\beta_{2} S E-a S, \\
E^{\prime}=\beta_{1} S I+\beta_{2} S E-b E, \\
I^{\prime}=\alpha E-c I .
\end{gathered}
$$

Therefore,

$$
\begin{array}{ll}
a=p+\mu, & b=k+\alpha+\mu, \\
c=r+\mu, & (1-p) N=A,
\end{array}
$$

where $N$ denotes the rate at which external computers are connected to the network; $p$ denotes the recovery rate of susceptible computer due to the anti-virus ability of network; $k$ denotes the recovery rate of exposed computer due to the anti-virus ability of network; $\beta_{1}$ denotes the rate at which, when having a connection to one infected computer, one susceptible computer can become exposed but has not broken-out; $\beta_{2}$ denotes the rate of which, when having connection to one exposed computer, one susceptible computer can become exposed; $\alpha$ denotes the rate of the exposed computers cannot be cured by anti-virus software and broken-out; $r$ denotes the recovery rate of infected computers that are cured; $\mu$ denotes the rate at which one computer is removed from the network. All the parameters are nonnegative.

Moreover, all feasible solutions of the system (3) are bounded and enter the region $D$, where

$$
D=\left\{(S, E, I) \in R_{+}^{3} \mid S \geq 0, E \geq 0, I \geq 0, S+E+I \leq \frac{A}{a}\right\} .
$$

Referring to [9], we define the basic reproduction number of the infection as

$$
R_{0}=\frac{A\left(\beta_{1} \alpha+\beta_{2} c\right)}{a b c} .
$$

For system (3), there always exists the virus-free equilibrium which is $P^{0}(A / a, 0,0)$; if $R_{0}>1$, then there also exists a viral equilibrium $P^{*}\left(S^{*}, E^{*}, I^{*}\right)$.

Therefore,

$$
\begin{gathered}
S^{*}=\frac{A}{a R_{0}}, \\
E^{*}=\frac{A\left(R_{0}-1\right)}{b R_{0}}, \\
I^{*}=\frac{A \alpha\left(R_{0}-1\right)}{b c R_{0}} .
\end{gathered}
$$

\section{The Virus-Free Equilibrium and Its Stability}

Theorem 1. $P^{0}$ is locally asymptotically stable if $R_{0}<1$. Whereas $P^{0}$ is unstable if $R_{0}>1$.

Proof. The characteristic equation of (3) at $P^{0}$ is given by

$$
\operatorname{det}\left(\begin{array}{ccc}
\lambda+a & -\beta_{2} S & -\beta_{1} S \\
0 & \lambda-\left(\beta_{2} S-b\right) & \beta_{1} S \\
0 & \alpha & \lambda+c
\end{array}\right)=0
$$

which equals to

$$
(\lambda+a)\left[\lambda^{2}-\left(\beta_{2} S^{0}-b-c\right) \lambda-b c\left(R_{0}-1\right)\right]=0 .
$$

Then, (9) has negative real part characteristic roots:

$$
\begin{gathered}
\lambda_{1}=-a, \\
\lambda_{2,3}=\frac{\left(\beta_{2} S-b-c\right) \pm \sqrt{\left(\beta_{2} S-b-c\right)^{2}+4 a b c\left(R_{0}-1\right)}}{2},
\end{gathered}
$$

where

$$
\beta_{2} S-b-c<0 \text {. }
$$


When $R_{0}<1$, there are no positive real roots of (9) and thus $P^{0}$ is a local asymptotically stable equilibrium. While $R_{0}>1$,(9) has positive real roots, which means $P^{0}$ is unstable.

The proof is completed.

Theorem 2. $P^{0}$ is globally asymptotically stable with respect to $D$ if $R_{0}<1$.

Proof. Let $L=\left(\left(\beta_{1} c+\beta_{2} \alpha\right) / b c\right) E+\beta_{2} I / c$.

Obviously

$$
L>0
$$

thus

$$
\begin{aligned}
L^{\prime}= & \frac{\left(\beta_{1} c+\beta_{2} \alpha\right)}{b c} E^{\prime}+\frac{\beta_{2}}{c} I^{\prime} \\
= & \frac{\left(\beta_{1} c+\beta_{2} \alpha\right)}{b c}\left(\beta_{1} S I+\beta_{2} S E-b E\right)+\frac{\beta_{2}}{c}(\alpha E-c I) \\
= & \frac{\left(\beta_{1} c+\beta_{2} \alpha\right)}{b c}\left(\beta_{1} I+\beta_{2} E\right) S-\frac{\left(\beta_{1} c+\beta_{2} \alpha\right)}{b c} b E \\
& +\frac{\beta_{2} \alpha E}{c}-\beta_{2} I \\
= & \frac{\left(\beta_{1} c+\beta_{2} \alpha\right)}{b c}\left(\beta_{1} I+\beta_{2} E\right) S-\beta_{1} E-\beta_{2} I \\
= & \left(\beta_{1} E+\beta_{2} I\right)\left[\frac{\left(\beta_{1} c+\beta_{2} \alpha\right)}{b c} S-1\right] \\
= & \left(\beta_{1} E+\beta_{2} I\right)\left[\frac{1}{b c} \cdot \frac{A}{a} \cdot \frac{R_{0} a b c}{A}-1\right] \\
= & \left(\beta_{1} E+\beta_{2} I\right)\left(R_{0}-1\right)<0 .
\end{aligned}
$$

The proof is completed.

\section{The Viral Equilibrium and Its Stability}

Theorem 3. $P^{*}$ is locally asymptotically stable if $R_{0}>1$.

Proof. The Jacobin matrix of system (3) about $P^{*}$ is given by

$$
J^{*}=\left(\begin{array}{ccc}
-a R_{0} & -\beta_{2} S^{*} & -\beta_{1} S^{*} \\
a\left(R_{0}-1\right) & \beta_{2} S^{*}-b & \beta_{1} S^{*} \\
0 & \alpha & -c
\end{array}\right),
$$

which equals to

$$
f(\lambda)=a_{0} \lambda^{3}+a_{1} \lambda^{2}+a_{2} \lambda+a_{3}=0,
$$

where

$$
\begin{gathered}
a_{0}=1, \\
a_{1}=a R_{0}-\left(\beta_{2} S^{*}-b-c\right)>0, \\
a_{2}=a b R_{0}+a c R_{0}-a \beta_{2} S^{*} \\
>a b R_{0}+a c R_{0}-a(b+c) \\
=a(b+c)\left(R_{0}-1\right)>0,
\end{gathered}
$$

where

$$
\beta_{2} S^{*}<b+c
$$$$
a_{3}=a b c\left(R_{0}-1\right)>0 \text {. }
$$

Thus,

$$
\begin{gathered}
\Delta_{1}=a_{1}>0, \\
\Delta_{2}=\left|\begin{array}{ll}
a_{1} & 1 \\
a_{3} & a_{2}
\end{array}\right|=a_{1} a_{2}-a_{3}>0, \\
\Delta_{3}=\left|\begin{array}{ccc}
a_{1} & 1 & 0 \\
a_{3} & a_{2} & a_{1} \\
0 & 0 & a_{3}
\end{array}\right|=a_{3}\left(a_{1} a_{2}-a_{3}\right)>0 .
\end{gathered}
$$

According to the Hurwitz criterion, all roots of (15) have negative real pats. Thus, the claimed result follows. The proof is completed. [9]).

The following result can be proved in the same way (see

Theorem 4. $P^{*}$ is uniquely globally asymptotically stable if $R_{0}>1$.

Proof. The Jacobin matrix of system (3) about $P^{*}$ is given by

$$
J^{*}=\left(\begin{array}{ccc}
-a R_{0} & -\beta_{2} S^{*} & -\beta_{1} S^{*} \\
a\left(R_{0}-1\right) & \beta_{2} S^{*}-b & \beta_{1} S^{*} \\
0 & \alpha & -c
\end{array}\right) .
$$

The second compound matrix $J^{[2]}$ of the Jacobin matrix can be calculated as follows (see $[10,11]$ ):

$$
J^{[2]}=\left(\begin{array}{ccc}
-a R_{0}+\beta_{2} S^{*}-b & \beta_{1} S^{*} & \beta_{1} S^{*} \\
\alpha & -a R_{0}-c & -\beta_{2} S^{*} \\
0 & a\left(R_{0}-1\right) & \beta_{2} S-b-c
\end{array}\right) .
$$

Set $P$ as the following diagonal matrix:

$$
P(x)=\left(1, \frac{E}{I}, \frac{E}{I}\right) .
$$

Denote that

$$
P_{f} P^{-1}=\operatorname{diag}\left(0, \frac{E^{\prime}}{E}-\frac{I^{\prime}}{I}, \frac{E^{\prime}}{E}-\frac{I^{\prime}}{I}\right) .
$$


Therefore, the matrix $B=P_{f} P^{-1}+P J^{[2]} P^{-1}$ can be written in the following block form:

$$
B=\left(\begin{array}{ll}
B_{11} & B_{12} \\
B_{21} & B_{22}
\end{array}\right)
$$

with

$$
\begin{gathered}
B_{11}=-a R_{0}+\beta_{2} S^{*}-b, \\
B_{12}=\frac{I}{E} \beta_{1} S^{*}(1,1), \\
B_{21}=\frac{E}{I}(\alpha, 0)^{T}, \\
B_{22}=\left(\begin{array}{cc}
\frac{E^{\prime}}{E}-\frac{I^{\prime}}{I}-\left(a R_{0}+c\right) & -\beta_{2} S^{*} \\
a\left(R_{0}-1\right) & \frac{E^{\prime}}{E}-\frac{I^{\prime}}{I}+\left(\beta_{2} S *-b-c\right)
\end{array}\right),
\end{gathered}
$$

thus

$$
\begin{gathered}
\mu_{1}\left(B_{11}\right)=\beta_{2} S^{*}-a R_{0}-b, \\
\mu_{1}\left(B_{22}\right)=\max \left\{\frac{E^{\prime}}{E}-\frac{I^{\prime}}{I}-a R_{0}-c+a R_{0}-a,\right. \\
\left.\quad \frac{E^{\prime}}{E}-\frac{I^{\prime}}{I}+\beta_{2} S *-b-c-\beta_{2} S^{*}\right\} \\
=\frac{E^{\prime}}{E}-\frac{I^{\prime}}{I}-c-a .
\end{gathered}
$$

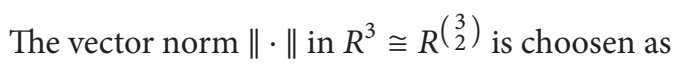

$$
\|(u, v, w)\|=\max \{|u|,|v+w|\} .
$$

The Lozinskii measure $\mu(B)$ with respect to $\|\cdot\|$ is as follows (see [12]):

$$
\mu(B) \leq \sup \left\{g_{1}, g_{2}\right\},
$$

where

$$
\begin{gathered}
g_{1}=\mu_{1}\left(B_{11}\right)+\left|B_{12}\right|=\beta_{2} S^{*}-a R_{0}-b+\frac{I}{E} \beta_{1} S^{*}, \\
g_{2}=\mu_{1}\left(B_{22}\right)+\left|B_{21}\right|=\frac{E^{\prime}}{E}-\frac{I^{\prime}}{I}-a-c+\frac{E}{I} \alpha .
\end{gathered}
$$

From (3), we find that

$$
\begin{aligned}
\frac{I}{E} \beta_{1} S^{*} & =\frac{E^{\prime}}{E}-\beta_{2} S^{*}+b, \\
\frac{I^{\prime}}{I} & =\frac{E}{I} \alpha-c,
\end{aligned}
$$

thus

$$
\begin{gathered}
g_{1}=\frac{E^{\prime}}{E}-a R_{0}, \\
g_{2}=\frac{E^{\prime}}{E}-a .
\end{gathered}
$$

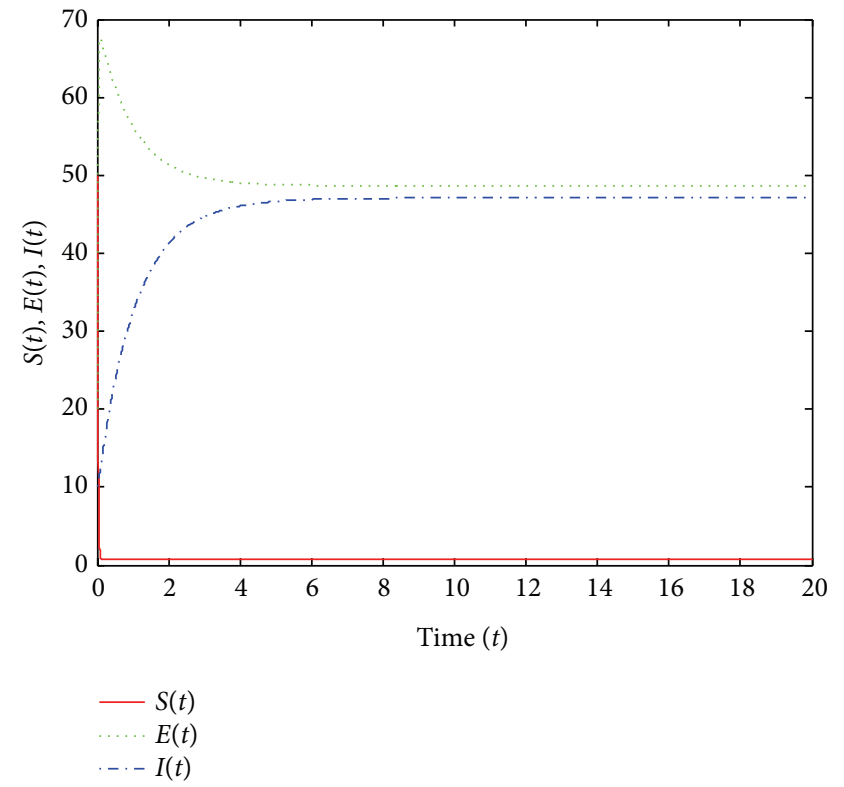

Figure 1: Dynamical behavior of system (3). Time series of susceptible, exposed, and infectious computers $S(t), E(t), I(t)$ with $R_{0}>1$.

Relations (28)-(30) imply that

$$
\mu(B) \leq \frac{E^{\prime}}{E}-a .
$$

Thus,

$$
\frac{1}{t} \int_{0}^{t} \mu(B) d \tau \leq \frac{1}{t} \int_{0}^{t}\left(\frac{E^{\prime}}{E}-a\right) d \tau=\frac{1}{t} \ln \frac{E(t)}{E(0)}-a .
$$

If $R_{0}>1$, then the virus-free equilibrium is unstable by Theorem 1. Moreover, the behavior of the local dynamic near $D_{0}$ as described in Theorem 1 implies that the system (3) is uniformly persistent in $D$; that is, there exists a constant $c_{1}>0$ and $T>0$, such that $t>T$ implies that

$$
\begin{gathered}
\lim _{t \rightarrow \infty} \inf S(t)>c_{1}, \\
\lim _{t \rightarrow \infty} \inf E(t)>c_{1}, \\
\lim _{t \rightarrow \infty} \inf I(t)>c_{1}, \\
\lim _{t \rightarrow \infty} \inf [1-S(t)-E(t)-I(t)]>c_{1} .
\end{gathered}
$$

For all $(S(0), E(0), I(0) \in D)($ see $[13,14])$,

$$
\bar{q}=\lim _{t \rightarrow \infty} \sup _{x \in K} \sup _{x \in K} \frac{1}{t} \int_{0}^{t} \mu(B) d \tau \leq-\frac{a}{2}<0 .
$$

The proof is complete.

\section{Numerical Examples}

For the system (3), Theorem 2 implies that the virus dies out if $R_{0}<1$, and Theorem 4 implies that the virus persists if $R_{0}>1$. Now, we present two numerical examples.

Let $p=0.5, \mu=0.02, k=0.4, \alpha=0.6, r=0.6, N=$ $100, \beta_{1}=0.7, \beta_{2}=0.8$, then $R_{0}=13.8>1$ and $\beta S^{*}<b+c$; Figure 1 shows the solution of system (3) when $R_{0}>1$. We 


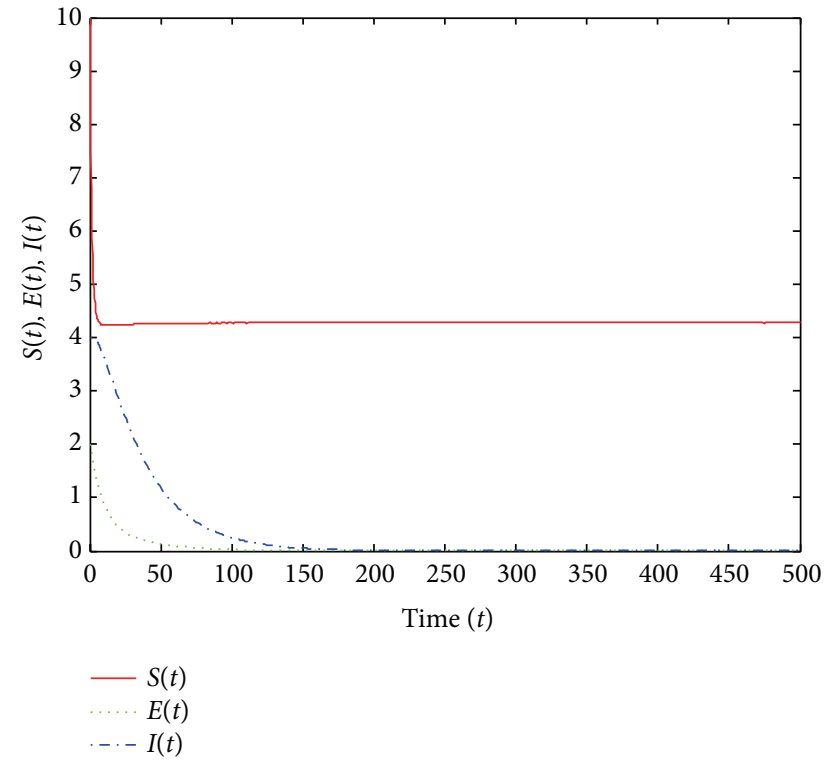

Figure 2: Dynamical behavior of system (3). Time series of susceptible, exposed, and infectious computers $S(t), E(t), I(t)$ with $R_{0}<1$.

can see that the viral equilibrium $P^{*}$ of system (3) is globally asymptotically stable.

Let $p=0.7, \mu=0.001, k=0.02, \alpha=0.09, r=$ $0.04, N=10, \beta_{1}=0.002, \beta_{2}=0.003$, then $R_{0}=0.1808<1$ and $\beta_{2} S^{0}<b+c$; Figure 2 shows the solution of system (3) when $R_{0}<1$. We can see that the virus-free equilibrium $P^{0}$ of the system (3) is globally asymptotically stable.

\section{Conclusion}

We assume that the virus process has a latent period and in these times the infected computers have infectivity also. A compartmental SEIR model for transmission of virus in computer network is formulated. In this paper, the dynamics of this model have been fully studied.

The results show that we should try our best to make $R_{0}$ less than 1 . The most effective way is to increase the parameters $p, k, r$ and decrease $\beta_{1}, \beta_{2}, \alpha$ and so on. Maybe in such way, the computer virus can be well predicted and thus controlled.

\section{Acknowledgment}

The work described in this paper was supported by the Science and Technology Project of Chongqing Education Committee under Grant KJ130519.

\section{References}

[1] C. Sun and Y.-H. Hsieh, "Global analysis of an SEIR model with varying population size and vaccination," Applied Mathematical Modelling, vol. 34, no. 10, pp. 2685-2697, 2010.

[2] L.-P. Song, Z. Jin, and G.-Q. Sun, "Modeling and analyzing of botnet interactions," Physica A, vol. 390, no. 2, pp. 347-358, 2011.
[3] J. Ren, X. Yang, L.-X. Yang, Y. Xu, and F. Yang, "A delayed computer virus propagation model and its dynamics," Chaos, Solitons \& Fractals, vol. 45, no. 1, pp. 74-79, 2012.

[4] B. K. Mishra and S. K. Pandey, "Dynamic model of worms with vertical transmission in computer network," Applied Mathematics and Computation, vol. 217, no. 21, pp. 8438-8446, 2011.

[5] X. Han and Q. Tan, "Dynamical behavior of computer virus on Internet," Applied Mathematics and Computation, vol. 217, no. 6, pp. 2520-2526, 2010.

[6] Q. Zhu, X. Yang, and J. Ren, "Modeling and analysis of the spread of computer virus," Communications in Nonlinear Science and Numerical Simulation, vol. 17, no. 12, pp. 5117-5124, 2012.

[7] L. X. Yang, X. Yang, Q. Zhu, and L. Wen, "A computer virus model with graded cure rates," Nonlinear Analysis: Real World Applications, vol. 14, no. 1, pp. 414-422, 2013.

[8] L. X. Yang, X. Yang, L. Wen, and J. Liu, "A novel computer virus propagation model and its dynamics," International Journal of Computer Mathematics, vol. 89, no. 17, pp. 2307-2314, 2012.

[9] P. van den Driessche and J. Watmough, "Reproduction numbers and sub-threshold endemic equilibria for compartmental models of disease transmission," Mathematical Biosciences, vol. 180, pp. 29-48, 2002.

[10] M. Fiedler, "Additive compound matrices and an inequality for eigenvalues of symmetric stochastic matrices," Czechoslovak Mathematical Journal, vol. 24(99), pp. 392-402, 1974.

[11] J.S. Muldowney, "Compound matrices and ordinary differential equations," The Rocky Mountain Journal of Mathematics, vol. 20, no. 4, pp. 857-872, 1990.

[12] G. Butler, H. I. Freedman, and P. Waltman, "Uniformly persistent systems," Proceedings of the American Mathematical Society, vol. 96, no. 3, pp. 425-430, 1986.

[13] H. I. Freedman, S. G. Ruan, and M. X. Tang, "Uniform persistence and flows near a closed positively invariant set," Journal of Dynamics and Differential Equations, vol. 6, no. 4, pp. 583600, 1994.

[14] P. Waltman, "A brief survey of persistence in dynamical systems," in Delay Differential Equations and Dynamical Systems (Claremont, CA, 1990), S. Busenberg and M. Martelli, Eds., vol. 1475, pp. 31-40, Springer, Berlin, Germany, 1991. 


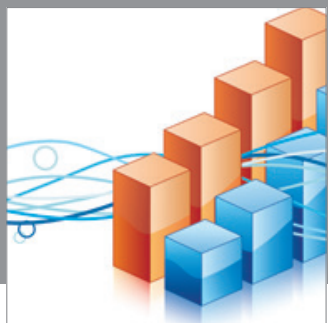

Advances in

Operations Research

mansans

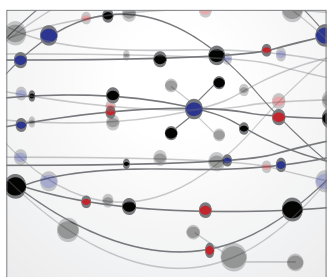

The Scientific World Journal
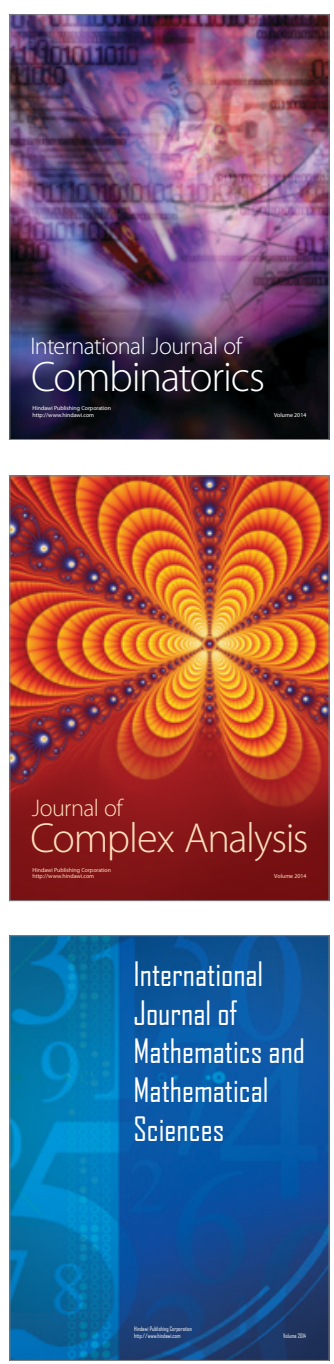
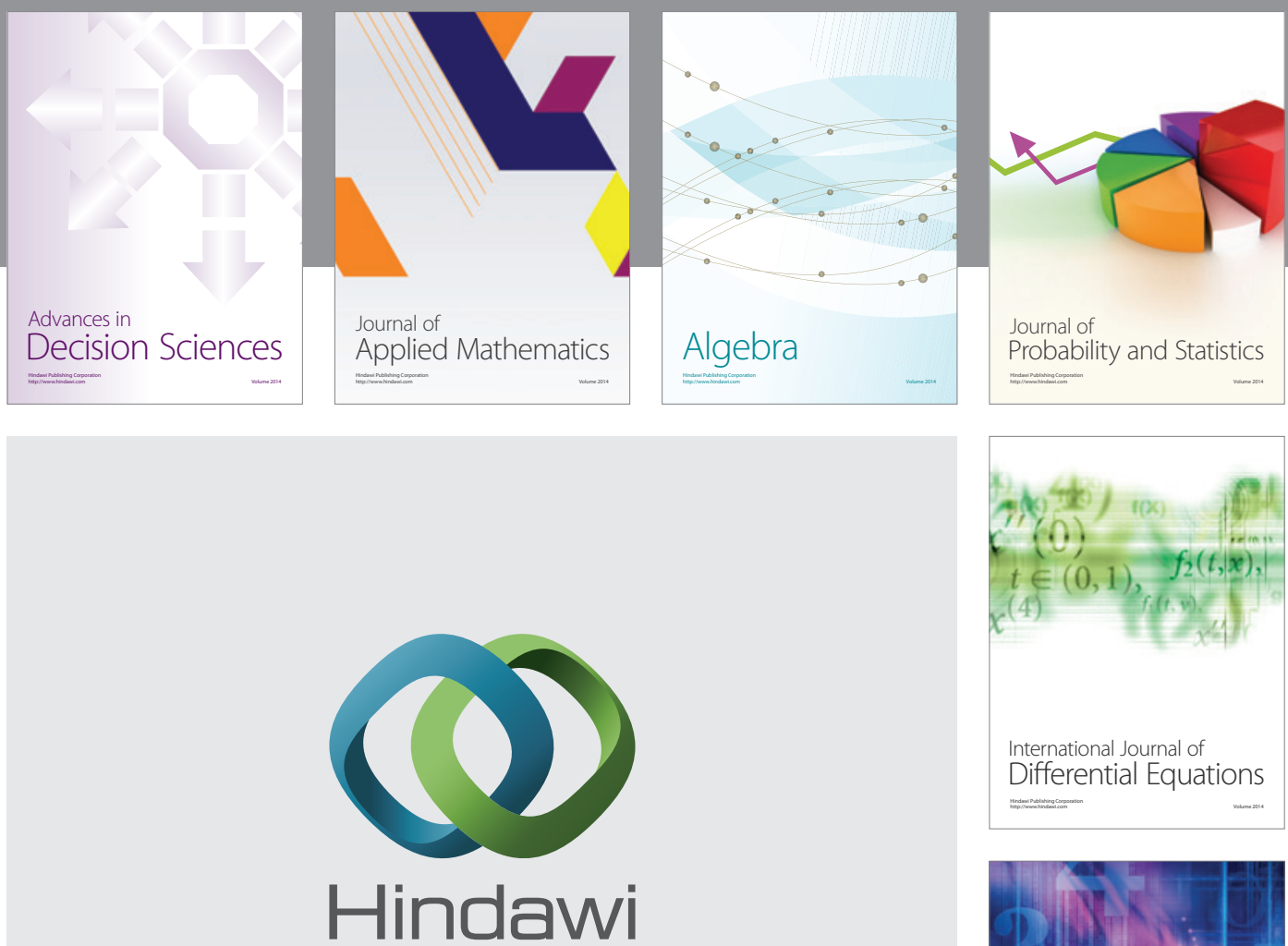

Submit your manuscripts at http://www.hindawi.com
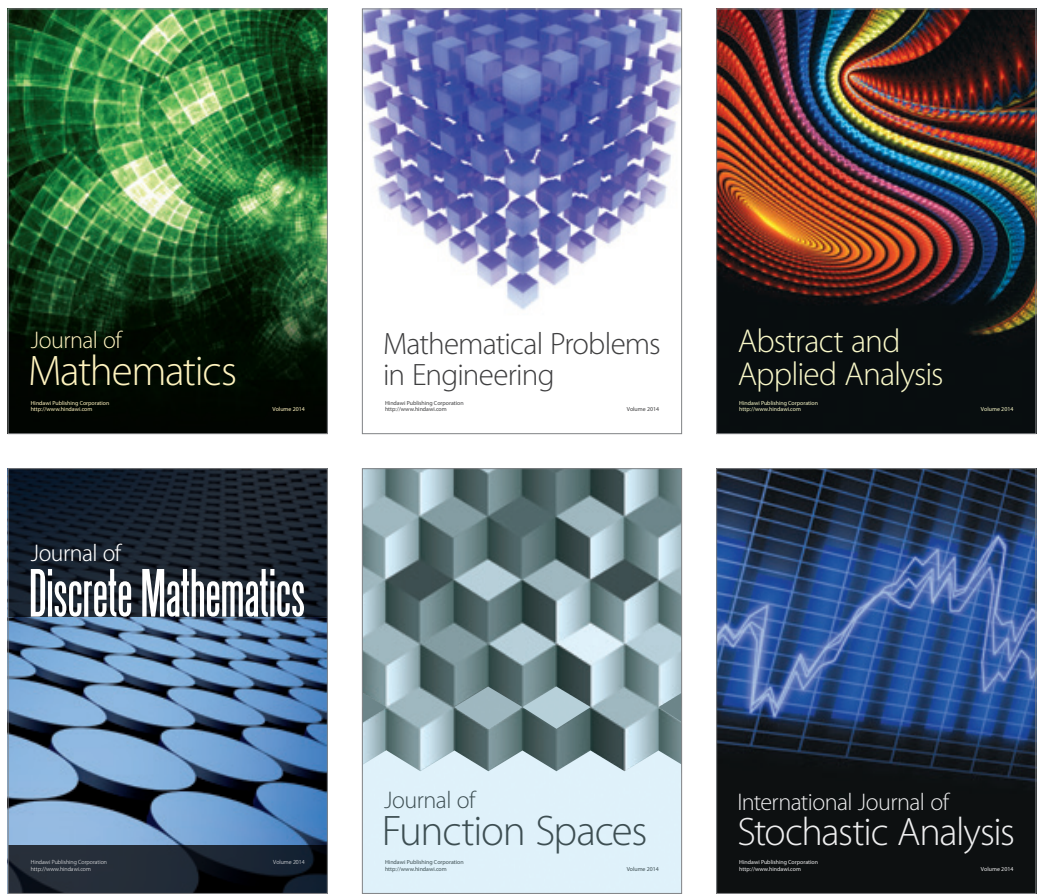

Journal of

Function Spaces

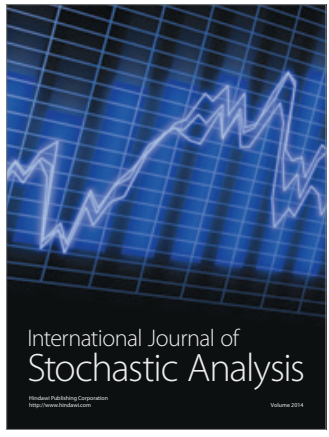

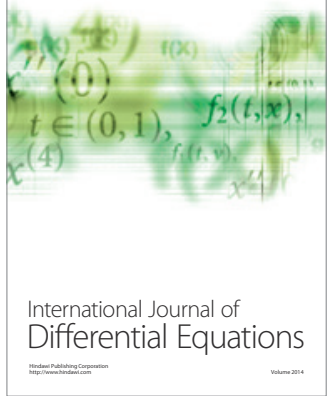
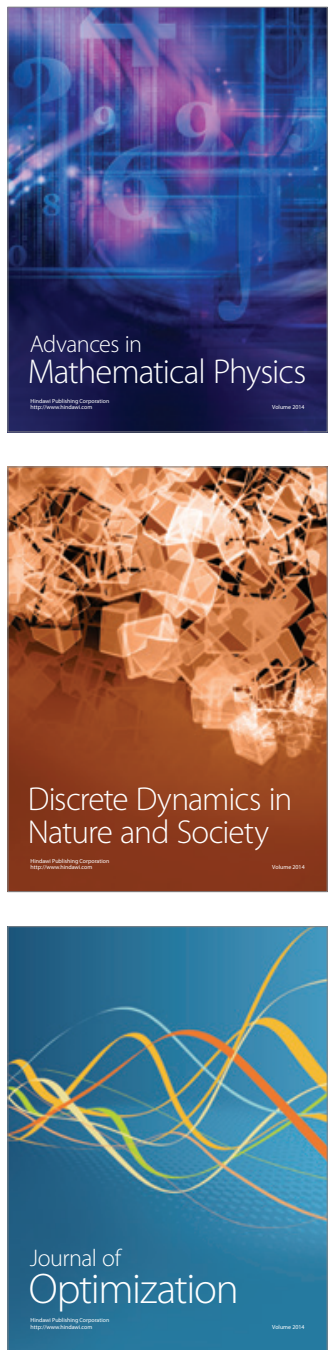